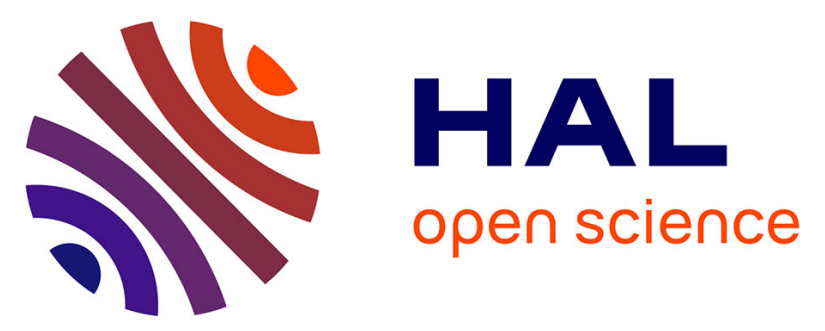

\title{
Influence of roughness on mechanical strength of direct bonded silica and Zerodur glasses
}

Natacha Cocheteau, Aurelien Maurel-Pantel, Frédéric Lebon, Frédéric

Mazerolle, Iulian Rosu, Sonia Ait-Zaid, Isabelle Savin de Larclause

\section{- To cite this version:}

Natacha Cocheteau, Aurelien Maurel-Pantel, Frédéric Lebon, Frédéric Mazerolle, Iulian Rosu, et al.. Influence of roughness on mechanical strength of direct bonded silica and Zerodur glasses. International Journal of Adhesion and Adhesives, 2016, 68, pp.87-94. 10.1016/j.ijadhadh.2016.02.006 . hal-01284059

\section{HAL Id: hal-01284059 \\ https://hal.science/hal-01284059}

Submitted on 7 Mar 2016

HAL is a multi-disciplinary open access archive for the deposit and dissemination of scientific research documents, whether they are published or not. The documents may come from teaching and research institutions in France or abroad, or from public or private research centers.
L'archive ouverte pluridisciplinaire $\mathbf{H A L}$, est destinée au dépôt et à la diffusion de documents scientifiques de niveau recherche, publiés ou non, émanant des établissements d'enseignement et de recherche français ou étrangers, des laboratoires publics ou privés. 


\section{Influence of roughness on mechanical strength of direct bonded Silica and Zerodur ${ }^{\circledR}$ glasses}

N. Cocheteau ${ }^{\text {a }}$, A. Maurel-Pantel ${ }^{\text {a }}$, F. Lebon ${ }^{\text {a }}$, F. Mazerolle ${ }^{\text {a }}$, I. Rosu ${ }^{\text {a }}$, S. Ait-Zaid ${ }^{\text {b }}$ I. Savin De Larclause ${ }^{b}$

${ }^{a}$ LMA, Mechanics and Acoustics Laboratory, CNRS, UPR 7051, Aix-Marseille Univ, Centrale Marseille, 31, Chemin Joseph Aiguier, F-13402 Marseille Cedex 20, France

${ }^{b}$ CNES, National Center for Spatial Studies, 18 Avenue Édouard Belin 31400 Toulouse, France

Corresponding author: Assistant Professor Aurélien Maurel-Pantel

Address: Laboratoire de Mécanique et d'Acoustique, 31 chemin J. Aiguier, 13402 Marseille cedex 20, France

Tel: + $33(0) 491164227$

Fax: + 33 (0) 491164481

Email: maurel@1ma.cnrs-mrs.fr 


\section{Influence of roughness on mechanical strength of direct bonded silica and Zerodur ${ }^{\circledR}$ glasses}

Direct bonding is of particular interest for optical system manufacturing for spatial application. This process requires very precise physical and chemical preparations of surface, especially roughness controls. Thus, this paper proposes to understand and discuss the roughness influence on mechanical strength and bonding energy of an elementary mechanical structure after a room temperature bonding and an annealing at optimal temperature. First, surfaces are characterised and roughness is measured. At the same time, the performances of fused silica and Zerodur ${ }^{\circledR}$ glasses are compared. Then interface properties are measured using double shear and wedge tests. At the end of the paper, a discussion, based on literature models, is proposed to explain the existence of an optimum of roughness put in evidence in experimental results.

Keywords: Direct bonding, Roughness, Double shear tests, Wedge tests, Bonding energy, Mechanical strength. 


\section{Introduction}

Direct bonding aims to join two surfaces without the use of any adhesive or additional material $[1,2]$. Two solids with well-polished flat surfaces, when brought into close proximity, locally attract to each other by Van der Waals or hydrogen bonds (bond between a hydrogen atom and a negative polarized atom such as oxygen atom) and adhere or bond. The main applications are on silicon-on-insulator devices. Silicon based sensor and actuators, electronics substrates are other examples of wafer bonding applications [2-5]. Note that for these usual applications the structure dimensions are quite small. Recently, this process was used in the manufacturing of high performance optical system for terrestrial application such as Fabry-Perot interferometers, prism assemblies, etc. For example, this bonding process has been used in the manufacture of the largest slicer ever used for the Multi Unit Spectroscopic Explorer [6] as related in Figure 1. In this case, the dimensions of the structure are quite large, mechanical solicitations and internal stresses are very different.

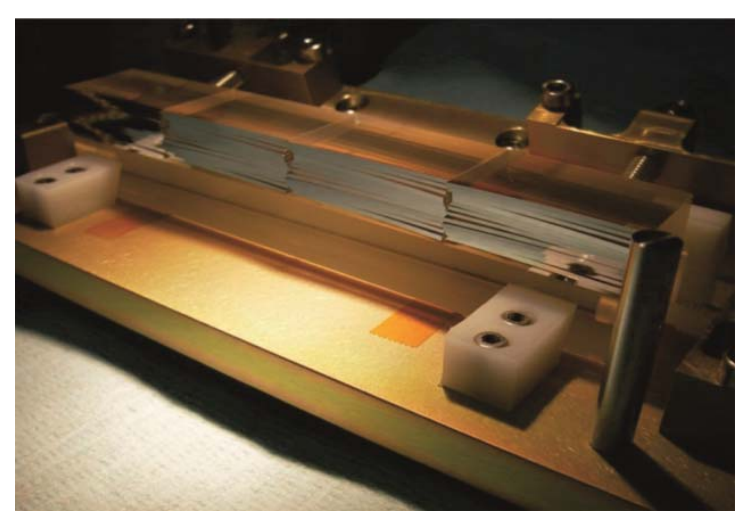

Figure 1: Slicer developed in the Multi Unit Spectroscopic Explorer (MUSE) for the Very Large Telescope (VLT) [6].

Nowadays, they are of particular interest for spatial instruments applications. Indeed, this is a high-precision production process, and assemblies obtained present a dimensional stability due to the absence of mechanical part or glue. In addition, since no adhesive materials are used in those processes, the risks of contamination associated with degassing are avoided, which is 
another advantage in spatial context. A first prototype has already passed with success the mechanical and thermal environment of space [7] where the constraints involved (thermal fatigue, accelerations, vibrations, etc.) are very different from those encountered on Earth. However, a better understanding of the assemblies' mechanical strength behaviour is required to validate the system life expectancy and to meet the European Space Agency standard. Mechanical strength of those bonded interfaces depends on the interface defects and the nature of bonds involved. Indeed, room temperature bonding needs flatness and roughness perfectly controlled, and no particles contaminations on surfaces [8-13]. Moreover, room temperature bonding is usually relatively weak; consequently, for some applications, the bonded assemblies undergo an annealing treatment causing changes in the nature of bonds responsible for adhesion and thus strengthen the bond across the interface $[14,15]$. Therefore, it is necessary to quantify the bonding strength and to improve the mechanical performance of adhesive bonds using the confidential Winlight Optics Company process. The first step of the process, the surface polishing, determines surface roughness and seems to be a crucial step in surface preparation of bonded elements $[9,12]$

Thus, in this paper it is proposed to discuss the roughness influence in direct bonding by quantifying mechanical strength and bonding energy evolution in function of surfaces, interfaces and process characteristics. The final aim is to understand mechanisms at the interface in order to find a way to determine an optimal roughness to obtain the best bonding in the assemblies.

First, fused silica glasses and Zerodur ${ }^{\circledR}$ glasses, which are specific materials used for spatial optical applications, are briefly presented as well as the sample preparation. At the beginning, the nature of both materials surfaces are compared using wetting tests and X-ray photoelectron spectroscopy analysis (XPS). Roughness is measured with a special device based on the Nomarski polarized light interferometry. 
Then the influence of roughness on the mechanical strength and the bonding energy at the interface of direct bonded silica and Zerodur interfaces after room temperature bonding with and without thermal treatment is investigated experimentally. The mechanical strength and the bonding energy of bonded interfaces is also compared for different roughness values using double shear experiments and wedge tests. Lastly, a discussion is developed to explain the existence of an optimal roughness giving a maximal bonding energy. In particular, a strategy is discussed based on two simple models available in literature describing roughness and spatial repartition at a nanoscale.

\section{Direct bonding process}

Direct bonding is possible as long as the two surfaces have similar geometries and requires clean surfaces free of contaminants $[10,16]$. Therefore, first steps of the Winlight Optics Company process consist in physical and chemical preparation of surfaces.

\subsection{Surface preparation}

Several polishing of both surfaces to adhere were manually performed in order to obtain the exact surface roughness (here, roughness is always less than $1 \mathrm{~nm}$ RMS), flatness and deflection required. Indeed, a high level of roughness results in a weak contact zone and thus in the occurrence of defects during the bonding process. When the roughness is too great, bonding becomes impossible [17-20]. Then, surfaces undergo a chemical treatment in order to eliminate any contaminating particles [21]. With ambient water, free silicon surfaces are recovered by silanol $(\mathrm{Si}-\mathrm{OH})$ groups which are the precursor of the bonding $[22,23,28]$. With this chemical treatment, the more silanol groups possible on both surfaces is seek. On figure 2, one component of the roughness is represented, each line represents a bonding site, and obviously a lack of bonding sites causes incomplete bonding. 
Wafer surfaces :

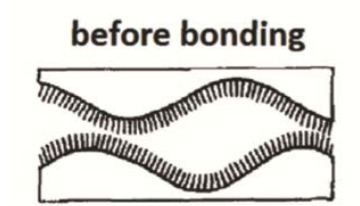

After spontaneous RT bonding

After RT bonding by

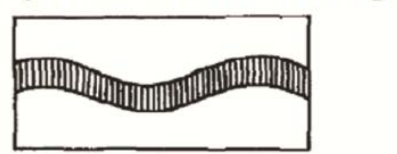

means of static pressure

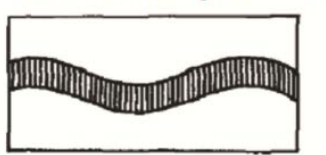

Fully hydrated surfaces

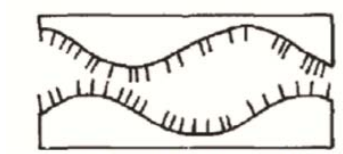

Poor local hydrophilization
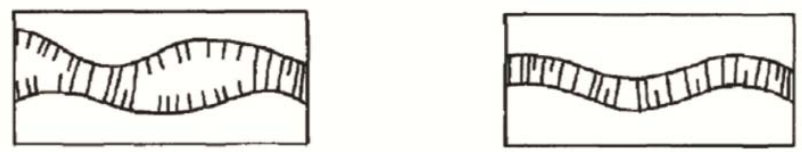

of surfaces

Figure 2: Behaviour of surfaces during room temperature bonding according to their

hydroxylated rate (from [14]).

\subsection{Room temperature bonding}

Once physical preparation and surface treatment has been completed, surfaces are brought into contact and bonding occurs between clusters of the two surfaces. By applying a slight local pressure, the surfaces are brought closer together, and hydrogen bonds developed between molecules of water present at the interface and the two surfaces. Molecules of water migrate from the bonding interface and/or diffuse at the interface, bringing the surfaces even closer together and initiating silanol-silanol covalent bonds [15, 24, 25].

\subsection{Evolution of bonded interfaces with temperature}

In order to obtain a stronger or irreversible sealing by increasing the number of covalent bonds, it is necessary to change the nature of bonds at the interface by applying for instance a thermal treatment [28]. Indeed, between $25^{\circ} \mathrm{C}$ and $200^{\circ} \mathrm{C}$, surfaces are contacted via clusters of waters $[14,15]$. When temperature is lower than $110^{\circ} \mathrm{C}$, chemical reactions at the bonded interface are the same than during room temperature bonding i.e. formation of hydrogen bonds between molecules of water and surfaces. When temperature is between $200^{\circ} \mathrm{C}$ and $700^{\circ} \mathrm{C}$; surfaces are 
directly contacted via double hydrogen bonds (Phase II) [26]. Up to $700^{\circ} \mathrm{C}$ the polymerization reaction is triggered, resulting in the formation of covalent siloxane bonds [14, 15].

\subsection{Materials for direct bonding}

In optical applications, three materials are mainly used: fused silica glasses, Zerodur ${ }^{\circledR}$, and Zinc Selenide ( $\mathrm{ZnSe})$. In this work, we chose to study two of them the fused silica glasses and Zerodur $^{\circledR}$ glasses which are the most commonly used in spatial optical applications with a reasonable cost [28].

\section{Surface characterisation}

To characterize samples surfaces, roughness has been measured for each sample tested in direct bonding assemblies. Moreover, wetting tests and X ray spectroscopy (XPS) were performed on silica and Zerodur surfaces of two kinds: never bonded surfaces and bonded then debonded surfaces [28].

\subsection{Roughness measurement}

Roughness is measured using the zygo new view device based on the Nomarski polarized light interferometry. An unpolarized light $(\lambda=612.5 \mathrm{~nm})$ is circularly polarized. The polarized light passes through a Wallaston prism and is separated into two rays polarized at $90^{\circ}$, the sampling ray and the reference ray. The passage through the sample induces a phase deviation. The rays are recombined using another Wallaston prism. The combination of the rays leads to interference, brightening or darkening the image at that point according to the optical path [29]. Figure 3 shows an example of roughness measurement result obtained on a silica sample.

\subsection{Wetting tests and X-Ray Spectroscopy}

In order to determine the surface energy of the both surfaces to adhere wetting tests are performed. The wetting test used four different liquids (deionised water, ethylene-glycol, 
benzyl alcohol, diiodo-methane) with a drop volume equal to $1 \mu \mathrm{L}$. The angle of contact was determined by approximating the drop profile using the software Drop Shape Analysis V.1.80. Results summarised in Table 1 shown that surface energy is greater for Zerodur ${ }^{\circledR}$ samples.

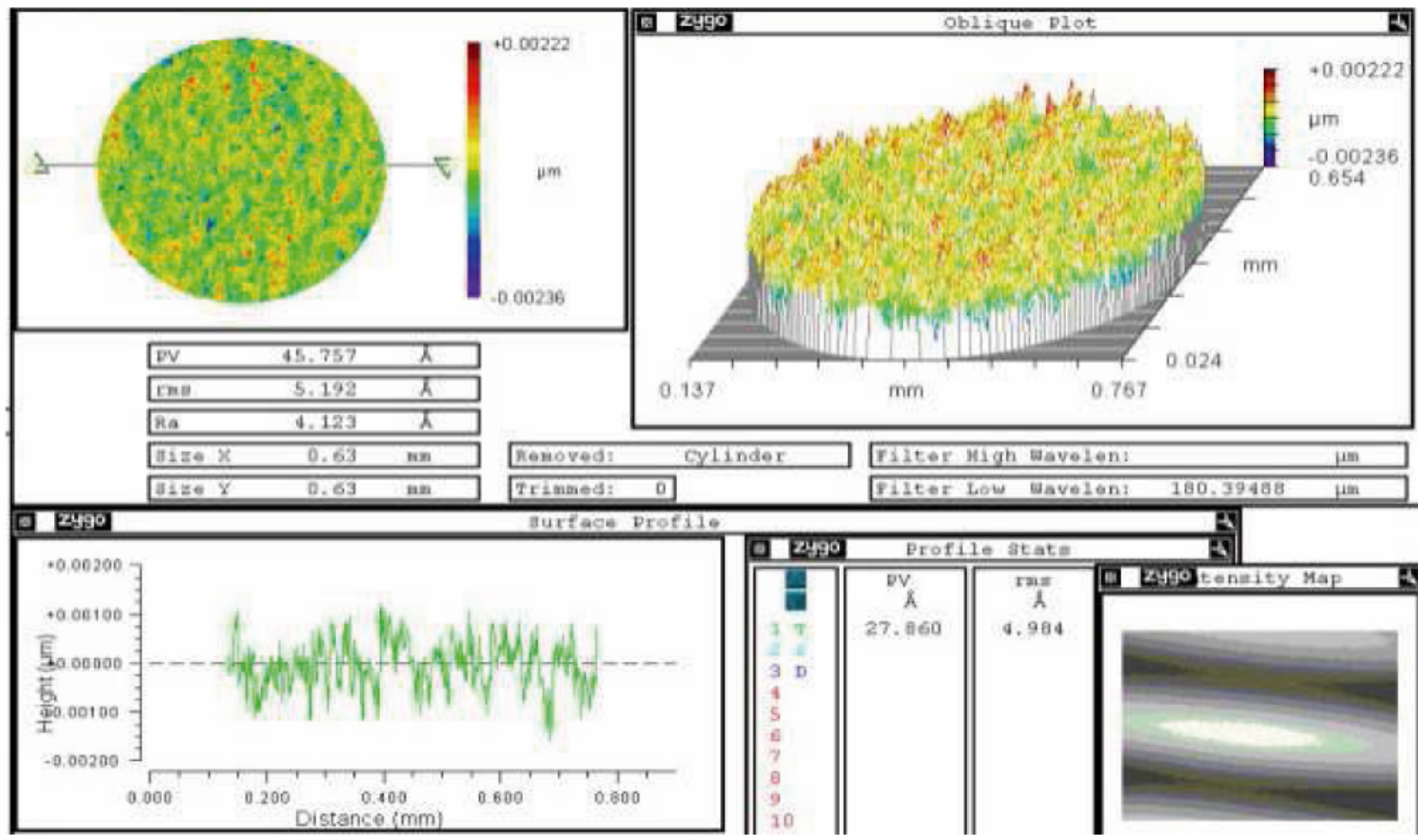

Figure 3: Roughness measurement results

Table 1: Surface energy for silica glasses and Zerodur glasses. [28]

\begin{tabular}{|l|l|}
\hline Material & $\gamma \mathrm{S}(\mathrm{mJ} / \mathrm{m} 2)$ \\
\hline Silica & 51,59 \\
\hline Debond Silica & 48,57 \\
\hline Zerodur & 40,43 \\
\hline Debond Zerodur & 41,52 \\
\hline
\end{tabular}

So this material seems to be a better candidate for direct bonding. According to the literature, the bonding will obviously be easier to perform when the surface energy of adhesion is high and furthermore when roughness is low [26], [12]. The difference in surface energy between non-bonded and debonded surfaces was found to be approximately $20 \%$. Surface wettability and XPS results show the quasi reversibility of the process [28] [30]. 


\section{Interface Characterisation}

To characterize assemblies' interfaces, it is proposed to measure mechanical strength and bonding energy of an elementary mechanical structure using a double shear test procedure and a wedge test. With these two mechanical tests, the influence of the roughness on two failure modes (I \& II) is observed.

\subsection{Double shear tests}

Double shear tests were performed to study the influence of the annealing parameters and roughness on the mechanical resistance in order to improve mechanical strength of bonded interfaces [28]. We assume that, in this case, the ultimate force is proportional to the bonding energy due to the brittle behaviour of molecular bonding. Table 2 shows the experiments performed to measure the influence of roughness ( 5 samples per tests).

Table 2: Summary of double shear tests performed [28].

\begin{tabular}{|c|c|}
\hline Materials & $\begin{array}{c}\text { Roughness } \\
\text { (nm RMS) }\end{array}$ \\
\hline Fused Silica & $0.2,0.4,0.6,1$ \\
\hline Zerodur $^{\circledR}$ & $0.2,0.6,1$ \\
\hline
\end{tabular}

\subsection{Wedge tests: Bonding energy measurement}

One of the main parameters classically used to characterize adhesion is the bonding energy [31], which can be connected to the critical strain energy release rate [8]. The most popular method to measure the bonding energy is the crack propagation method or wedge test. A razor blade is inserted at the interface between two blades bonded together. A crack will appear along the bonded interface until the establishment of equilibrium between the elasticity of blades and the bonds responsible for the adhesions which are hydrogen bonds in our case. The length of the crack 1 is measured with a camera using interference fringe due to the small thickness of air trapped at the open interface. 


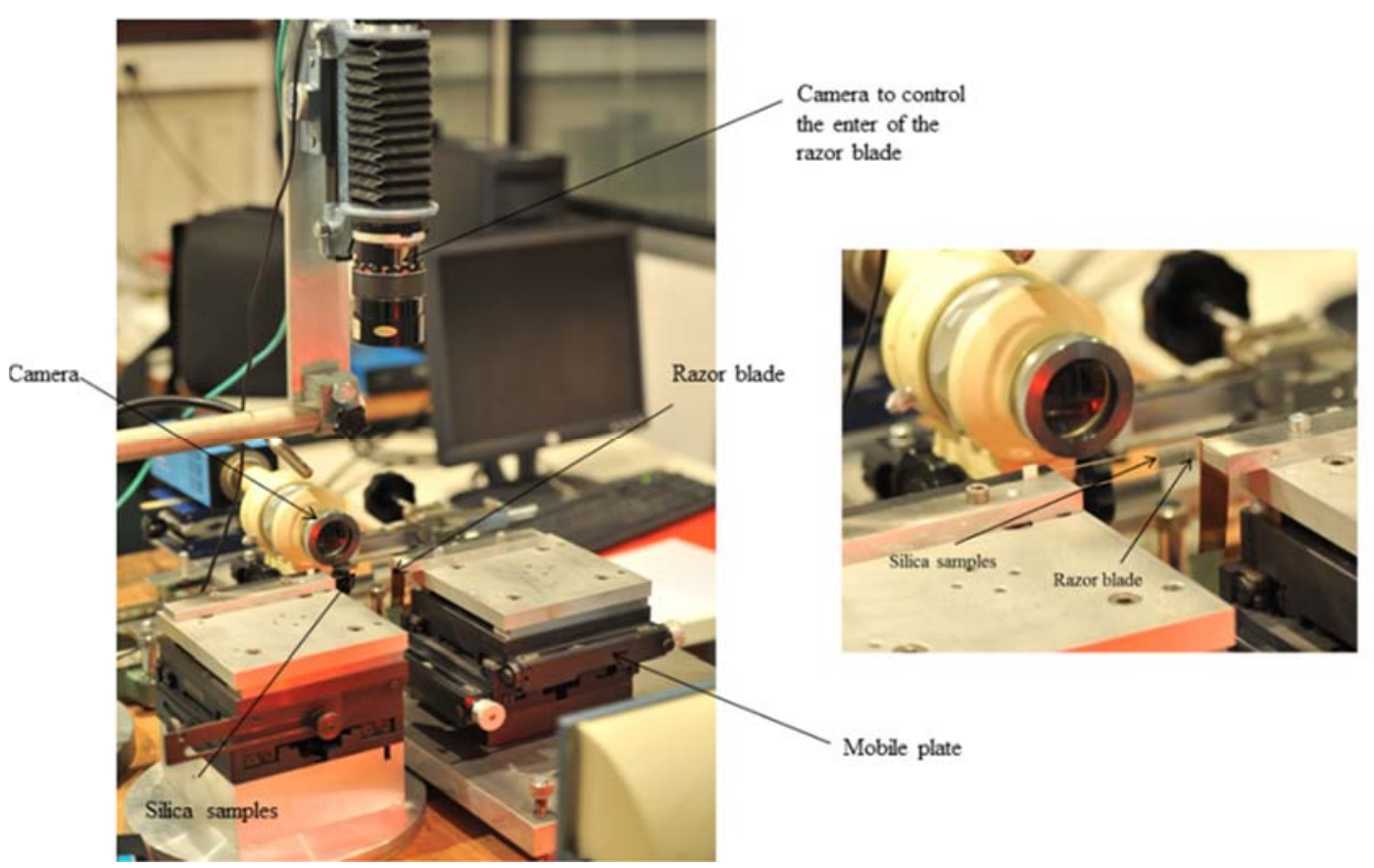

Figure 4: Experimental device of wedge test developed

At the equilibrium, the critical strain energy release rate is equal to the work of adhesion [3234] and related to the crack length using linear elastic fracture mechanics. When both surfaces are identical:

$\mathrm{G}_{1 \mathrm{C}}=\mathrm{W}_{\text {adhesion }}=2 \gamma$

With $\gamma$ the surface energy of surfaces. The critical strain energy release rate can be approximately related to the length 1 using the following equation [32]:

$\mathrm{G}_{1 \mathrm{C}}=\frac{3 \mathrm{Et}^{3} \mathrm{y}^{2}}{8 \mathrm{l}^{4}}$

Where $\mathrm{E}$ is the Young modulus of blades, $\mathrm{t}$ the blade thickness, $\mathrm{y}$ the razor blade thickness and 1 the length of the crack.

Samples are constituted with two blades of silica with $500 \mu \mathrm{m}$ of thickness $(\mathrm{t}), 10 \mathrm{~mm}$ of width and $80 \mathrm{~mm}$ of length bonded together. The razor blade $(100 \mu \mathrm{m}(\mathrm{y})$ of thickness) is always inserted by the same length using a mobile plate and the insertion is controlled with a camera above the blades as shown in Figure 4. Wedge test has been performed to study the evolution 
of the bonding energy with annealing parameters and roughness. Table 3 relates the experiments performed, two samples have been tested for each parameter twice. Between each tests, surfaces have been cleaned and re-adhered without being polished anew. Thermal treatments were limited to $200^{\circ} \mathrm{C}$ due to the results of the first study [28] and due to the optical and coating industrial constraints.

Table 3: Summary of wedge tests performed on fused silica samples

\begin{tabular}{|c|c|c|}
\hline $\begin{array}{c}\text { Annealing temperature } \\
\left({ }^{\circ} \mathrm{C}\right)\end{array}$ & $\begin{array}{c}\text { Annealing time } \\
(\mathrm{h})\end{array}$ & $\begin{array}{c}\text { Roughness } \\
(\mathrm{nm} \text { RMS })\end{array}$ \\
\hline- & - & $0.4,0.8$ \\
\hline 100 & $5,35,120$ & $0.4,0.8$ \\
\hline 200 & $5,35,120$ & $0.4,0.8$ \\
\hline
\end{tabular}

\subsection{Results and discussion}

Results presented in figure 5 show the evolution of the ultimate force with roughness coupled with thermal treatment $\left(200^{\circ}\right.$ during $120 \mathrm{~h}$ for silica samples and $130^{\circ}$ during $120 \mathrm{~h}$ for Zerodur samples). The roughness is measured using the experimental device described in Section 4 . We observe that the ultimate force increases with roughness until $0.6 \mathrm{~nm}$ RMS then decreases. But due to the dispersion, it seems important to check the validity of this statement with a statistical analysis (f-test and t-test types). In Zerodur case, we can't validate this assumption, it seems more plausible to conclude that all roughness give the same result (in term of mechanical strength all roughness are equivalent). In silicate case, the assumption is validated and moreover it seems that there is no difference between $0.6 \mathrm{~nm}$ and $1 \mathrm{~nm}$ RMS. So, in double shearing test, it seems that high roughness gives a best mechanical strength.

Figure 6 presents the evolution of the bonding energy with roughness; full line represents results for the first adhesion and the dotted line for the second adhesion. Results show the same trends, a higher bonding energy with an optimal roughness, which is not the lower one. Results also show a decrease of the bonding energy for the second adhesion highlighting a phenomenon of 
surface damaging with successive adhesion - wedge tests - re-adhesion. Those results suggest that for our physical and chemical preparation, a quite higher roughness is preferable. Too low roughness leads to the appearance of residual stresses on surfaces during polishing process. Moreover we have seen before that between $150^{\circ} \mathrm{C}$ and $450^{\circ} \mathrm{C}$ the bonding energy is limited by the contacted area which is higher for surfaces with higher roughness explaining better results for high roughness [28]. This phenomenon increases with thermal treatment; indeed, thermal treatment promotes the formation of siloxane covalent bonds [22] and thus increases the mechanical resistance and the bonding energy. In wafer bonding field, it is commonly accepted that the roughness minimal value gives the best bonding energy. But the experimental investigations performed and related results confirm the existence of a roughness optimal value in direct bonding strength improvement of fused silica glasses or Zerodur ${ }^{\circledR}$ assemblies.
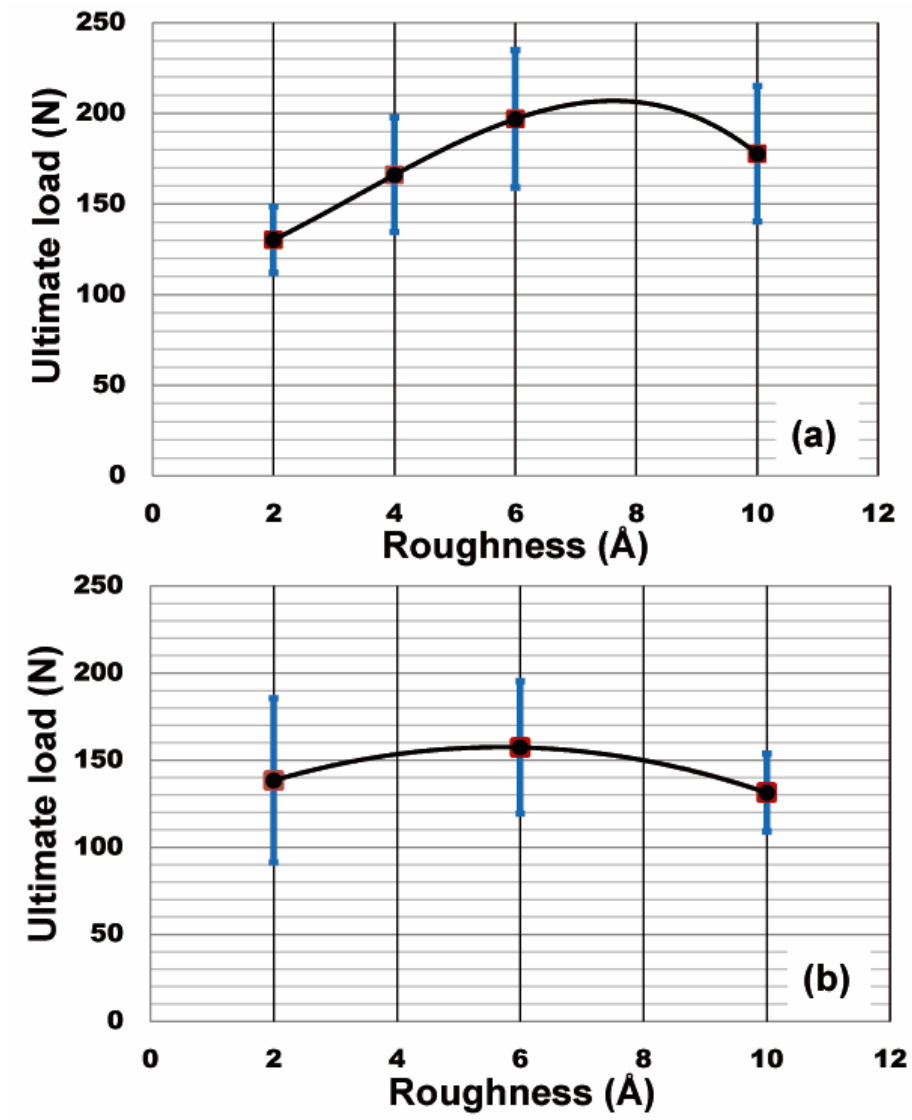

Figure 5: Roughness influence combined with thermal treatment on fused silica glass (a) and Zerodur ${ }^{\mathbb{B}}$ samples (b) tested in double shear configuration: average values and standard deviations of measured ultimate loads [28]. 


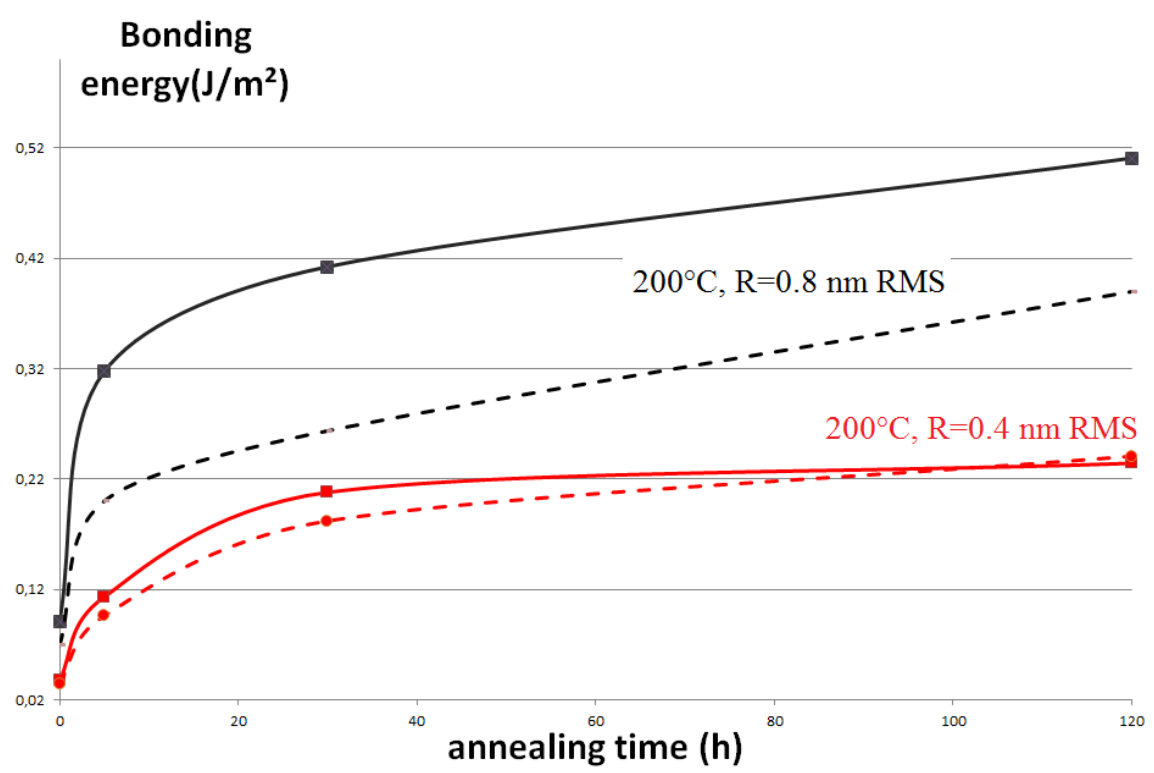

Figure 6: Influence of roughness measured with wedge tests on fused silica samples

\section{Discussion on roughness effect for room temperature bonding physics}

In literature the experimental investigation on direct bonding has shown the importance of roughness at room temperature contacting step. Indeed, if the roughness of the two surfaces is too large, adhesion became impossible. In this section, we proposed a discussion on roughness effect for room temperature bonding using two different simplified models available in the literature (one based on JKR theory and the other based on adhesion description at nanoscale) in order to understand and explain the existence of the roughness optimal value observed in experimental results.

\subsection{A simplified model of roughness: Tang et al. based on JKR theory}

A first simplified model based on JKR theory of the effect of roughness, due to Tang et al. [17] is recalled. By modeling the contact of two rough surfaces by the contact of one surface with the combine roughness and the other as a rigidly flat surface (Figure 7) it is possible to have a parameter - denoted $\alpha$ in the following - characterizing the bonding: spontaneous bonding, bonding using an external force or impossibility of bonding. 
(a)

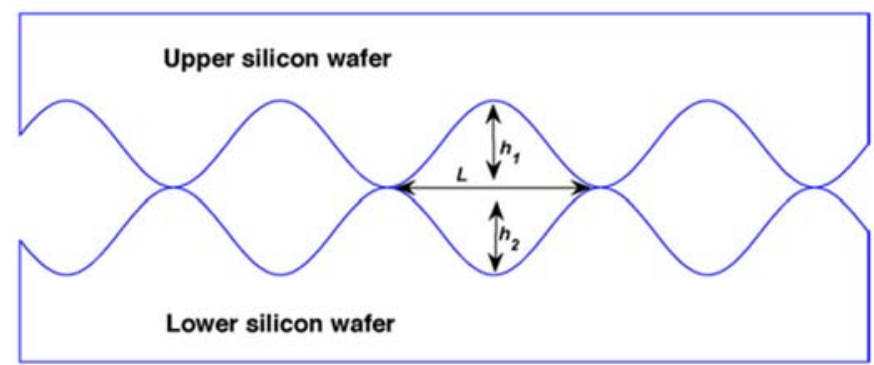

(b)

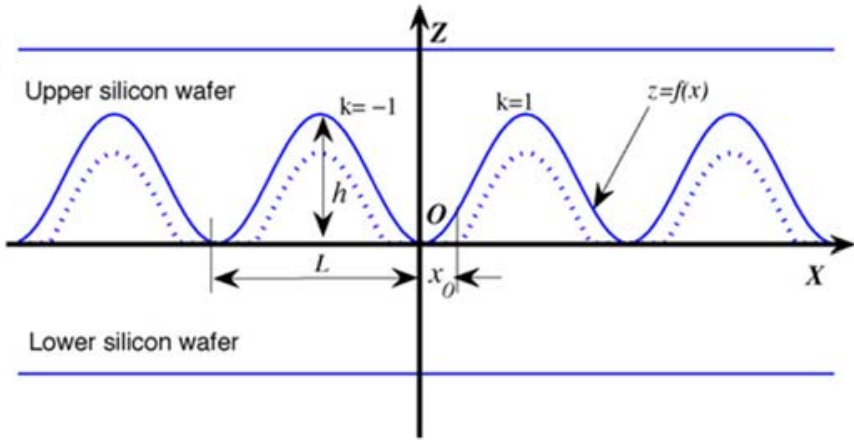

Figure 7: Schematic model of direct bonding for a periodic distribution of roughness (a) equivalent model. The solid line indicates the interface before bonding; dotted line represents the interface during bonding from [26]

The simplified cross sectional surface profile of the upper surface in the equivalent model (Figure 7) is:

$f(x)=\frac{L^{2}}{2 R \pi} \sin ^{2}\left(\frac{\pi x}{L}\right), x \in\left(k L-x_{0} ; k L+x_{0}\right), \quad 2 x_{0} \leq L$

Where $k$ is an integer, $x_{0}$ is the length of the contacted zones, $L$ the periodic length of the asperities (wavelength) and $h=\frac{L^{2}}{2 R \pi}$ the combine maximum height of the asperities with $R$ the mean asperities radius. After derivation detailed in Appendix (A), we can write the following equation:

$\widehat{F}=\sin ^{2}(\pi c)-\alpha \sqrt{\tan (\pi c)}$

Where:

$c=\frac{x_{0}}{L}$ 
And

$\alpha=4 \sqrt{2} \pi \sqrt{\frac{\omega R^{2}}{E^{*} L^{3}}}=\frac{2 \sqrt{2}}{\pi} \sqrt{\frac{\omega}{E^{*}} \cdot \frac{L}{h^{2}}}$

$\hat{F}$ is the force per asperity on the silicon surface contributed by both the adhesion force and the external force [15]. The first term corresponds to the normalized Hertz pressure and the second expresses the influence of the silicon surface force on the contact zone [26], [17], [18].

The dimensionless parameter $\alpha$ defines the state of bonding at room temperature: when $\alpha$ is inferior to 0.57 bonding is spontaneous, when $\alpha$ is between 0.57 and 1.065 bonding is possible with an external force and when $\alpha$ is superior to 1.065 bonding is impossible.

This parameter $\alpha$ traduces the importance of roughness on direct bonding at room temperature. Once the two surfaces are adhered it is important to study the influence of the roughness on the mechanical resistance and bonding energy. Figure 8 shows the evolution of the normalized contact force versus the normalized contact length $c$ for different values of $\alpha$. The contact force strongly depends on the roughness and mechanical parameters (height of roughness, wavelength, stiffness, etc.) at room temperature. Thus one way to improve the contact force is to increase the length of contacted zone, even if it is difficult to control in practice.

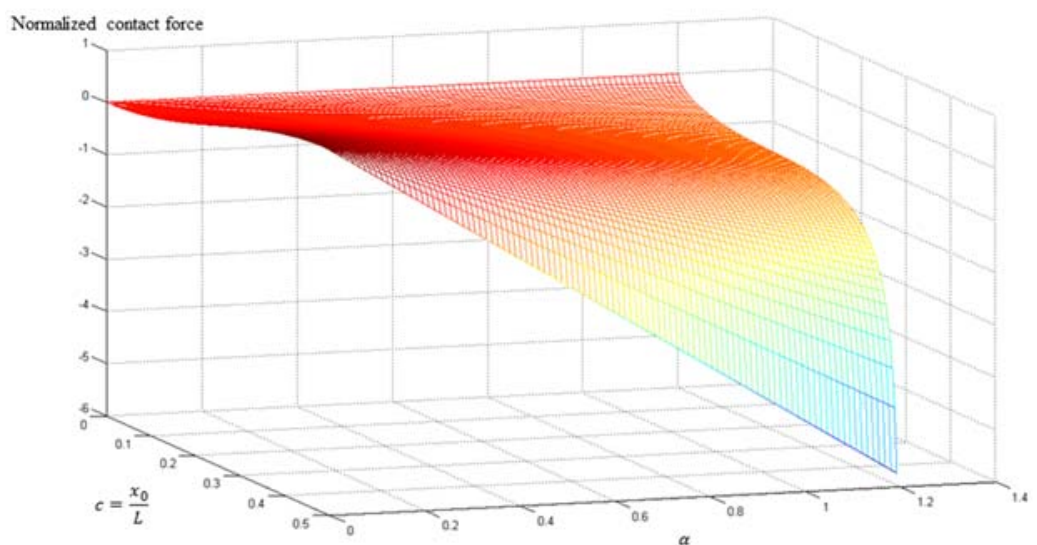

Figure 8: Evolution of the normalized contact force versus the normalized contact length $c$ for different values of $\alpha$ 
In this simplified model presented above (eq. 4) the adhesion depends on the height of the asperities, the curvature of the asperities, the wavelength, the real surface of contact and the local stiffness of the contact surfaces. Thus, the optimal roughness is necessarily a compromise between all these physical parameters. As a first step in this modelling, the coefficient $\alpha$ depends on the ratio between the height of the asperities and the wave length. For instance a simultaneous decrease or increase of these two parameters leads to the same bonding quality. Moreover, this model neglects residual stress due to the polishing process. In this modelling, it is possible to propose an analogy between the increase of residual stresses and the increase of the equivalent modulus $E^{*}$. Note, that the decrease of the bonding energy after a first bonding (damaging phenomenon), as observed in the experimental results obtained can be explained by an increase of the residual stresses due to polishing operations.

\subsection{A simplified model of roughness: adhesion at nanoscale}

Two small elements of surface with dimension $d s$ are considered. The interaction between these two surfaces is derived from the Lennard-Jones potential as proposed by Hamaker [36]. If the two surfaces are plane, the normal force density $T$ is given by:

$\mathrm{T}=\frac{\mathrm{A}}{3 \pi \mathrm{d}_{\mathrm{c}}^{3}}\left[\left(\frac{\mathrm{d}_{\mathrm{c}}}{\mathrm{d}}\right)^{3}-\left(\frac{\mathrm{d}_{\mathrm{c}}}{\mathrm{d}}\right)^{9}\right] \mathrm{Z}$

where $d$ is the distance between the two surfaces, $d_{c}$ is the critical distance (distance at equilibrium), $n$ the normal unit vector to the surface, and A a given constant. Hamaker defines the surface energy or energy of adhesion by the work necessary to separate the two surfaces i.e.:

$\mathrm{w}=\int_{\mathrm{d}_{\mathrm{c}}}^{+\infty} \frac{\mathrm{A}}{3 \pi \mathrm{d}_{\mathrm{c}}^{3}}\left[\left(\frac{\mathrm{d}_{\mathrm{c}}}{\mathrm{d}}\right)^{3}-\left(\frac{\mathrm{d}_{\mathrm{c}}}{\mathrm{d}}\right)^{9}\right] \mathrm{dl} \mathrm{ds}$ 
Thus,

$\mathrm{w}=\frac{\mathrm{Ands}}{8 \pi \mathrm{d}_{\mathrm{c}}^{2}}=\overline{\mathrm{w}} \mathrm{ds}$

Now, a simplified model of roughness is considered (see Figure 9), with only one asperity.

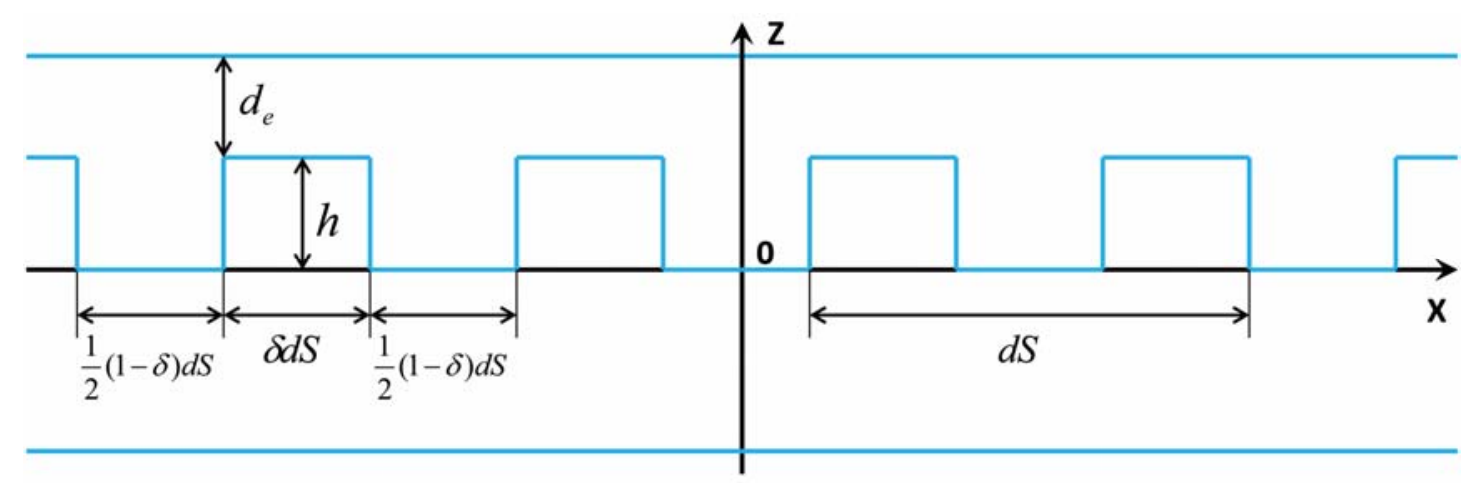

Figure 9: Schematic model of direct bonding for a periodic distribution of roughness (a) equivalent model.a simplified model of roughness

In the following, $h$ denotes the height of the asperity and $\delta d S$ its width. In this case, the normal force density is given by :

$\mathrm{T}=\frac{\mathrm{A}}{6 \pi \mathrm{d}_{\mathrm{c}}^{3}}\left[\delta\left(\frac{\mathrm{d}_{\mathrm{c}}}{\mathrm{d}}\right)^{3}-\delta\left(\frac{\mathrm{d}_{\mathrm{c}}}{\mathrm{d}}\right)^{9}+(1-\delta)\left(\frac{\mathrm{d}_{\mathrm{c}}}{\mathrm{d}+\mathrm{h}}\right)^{3}-(1-\delta)\left(\frac{\mathrm{d}_{\mathrm{c}}}{\mathrm{d}+\mathrm{h}}\right)^{9}\right] \mathrm{z}$

The equilibrium distance between the two surfaces and denoted by $d_{e}$ is given by the following equation:

$\delta\left(\frac{\mathrm{d}_{\mathrm{c}}}{\mathrm{d}_{\mathrm{e}}}\right)^{3}-\delta\left(\frac{\mathrm{d}_{\mathrm{c}}}{\mathrm{d}_{\mathrm{e}}}\right)^{9}+(1-\delta)\left(\frac{\mathrm{d}_{\mathrm{c}}}{\mathrm{d}_{\mathrm{e}}+\mathrm{h}}\right)^{3}-(1-\delta)\left(\frac{\mathrm{d}_{\mathrm{c}}}{\mathrm{d}_{\mathrm{e}}+\mathrm{h}}\right)^{9}=0$

Note that $d_{e} \leq d_{c}$. Obviously, the value $d_{e}$ can be found numerically using for instance a Newton method. Following the idea of Hamaker, the surface energy density is given by:

$\bar{w}=\int_{d_{e}}^{+\infty} \frac{A}{3 \pi d_{c}^{3}}\left[\delta\left(\frac{d_{c}}{d}\right)^{3}-\delta\left(\frac{d_{c}}{d}\right)^{9}+(1-\delta)\left(\frac{d_{c}}{d+h}\right)^{3}-(1-\delta)\left(\frac{d_{c}}{d+h}\right)^{9}\right] d l$ 
Thus,

$\overline{\mathrm{W}}=\frac{\mathrm{A}}{6 \pi}\left[\frac{\delta}{2 \mathrm{~d}_{\mathrm{e}}^{2}}-\frac{\delta \mathrm{d}_{\mathrm{c}}^{6}}{8 \mathrm{~d}_{\mathrm{e}}^{8}}+\frac{1-\delta}{2\left(\mathrm{~d}_{\mathrm{e}}+\mathrm{h}\right)^{2}}-\frac{(1-\delta) \mathrm{d}_{\mathrm{c}}^{6}}{8\left(\mathrm{~d}_{\mathrm{e}}+\mathrm{h}\right)^{8}}\right]$

On Figure 10, it is shown the surface energy density (with $A=1$ in order to simplify) as a function of the ratio between the length of the asperity and the critical distance (i.e. $\frac{h}{d_{c}}$ ), for various values of $\delta d S$.

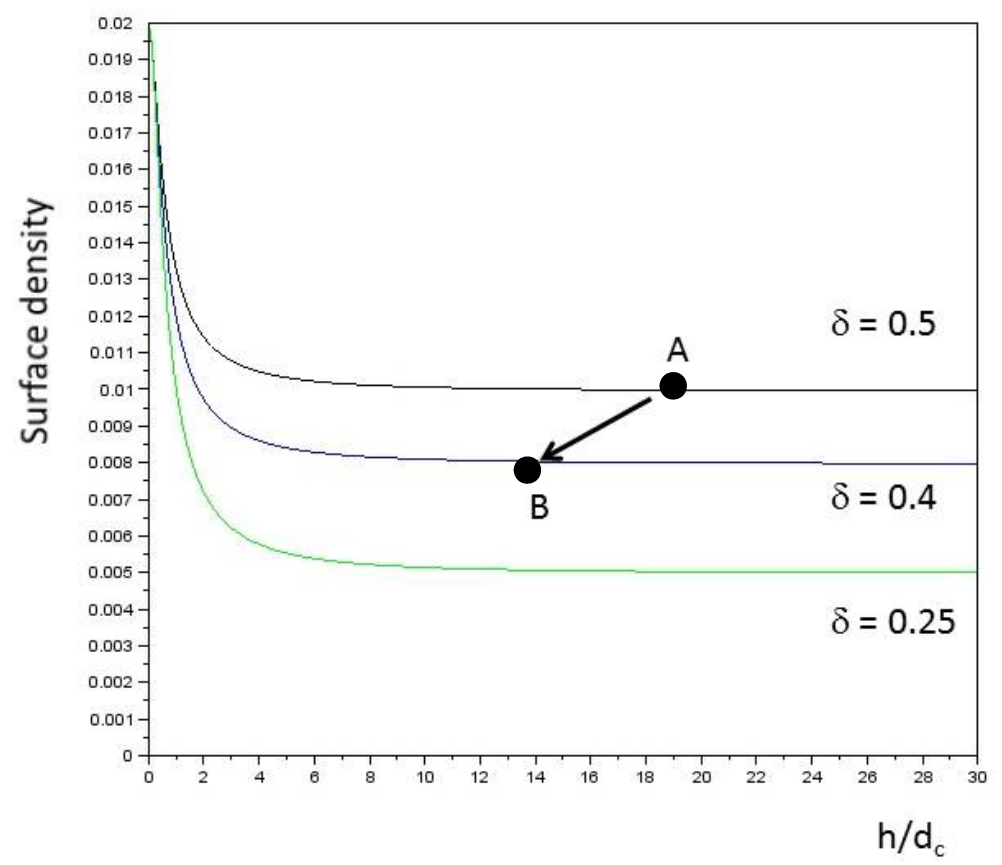

Figure 10: surface energy density

On this figure, it is observed that the behavior of the energy is strongly nonlinear. For a given value of the asperity width, the energy increases with the decrease of the roughness. In the same way, for a given value of the height of the roughness, the energy increases when the width of the asperity increases.

However, it can be observed that the conjugate effects, a simultaneous decrease width and height, can lead to a decrease of the energy (from point A to point B on the Figure 10). Thus, this simple model shows that in practice, to obtain a systematic increase of the bonding energy 
using polishing techniques (which lead to a decrease of the height of the asperities), it is necessary to control simultaneously the width of asperities.

Since the polishing process was done, with a fixed abrasive grain, it is possible that the height of the asperities was reduced while maintaining the same length at the minimal value of abrasive grain. This effect can partially explain the experimental results obtained in the previous section of this paper.

\section{Conclusion}

Roughness is an important parameter during room temperature direct bonding, characterizing the ability to adhere two surfaces. The literature for silicon wafer bonding relates that the minimal roughness gives the maximal bonding energy. These works proposed to make some experiments on elementary mechanical structure after a room temperature direct bonding and an annealing at optimal temperature in order to quantify the roughness influence on mechanical strength and bonding energy. So double shear tests and wedge tests were performed on fused silica glasses and Zerodur samples and show an optimal value of roughness to increase mechanical strength and bonding energy.

Two different simplified models were proposed and compared, to understand the existence of an optimal roughness. Each model relates physical mechanisms based on JKR theory and Lennard-Jones Potential. With these analogies, different ways can be imagined to understand the role of the roughness in direct bonding physical mechanisms. In both models, direct bonding mechanisms is a compromise between height and width evolutions of the asperities during the polishing process. Moreover, in the model based on JKR theory, direct bonding mechanisms is also a compromise between residual stresses due to low roughness and the size of the contacted zone. The roughness influence on mechanical strength, in glass elementary mechanical structure field, seems to be not only a problem of minimisation but about how to control and 
optimise the geometries of the asperities and the surface residual stresses.

In the future, we intend to propose a mathematical and numerical modeling of the direct bonding taking into account roughness characteristics (spatial repartition, amplitude, standard deviation,...). The final aim consists in the development of an interface mechanical model of direct bonding. The model should be an implemented law linking the bonding energy, the mechanical critical strain energy, the process parameters and the chemical kinetic in a multiphysic and multi-scale formalism in order to help to design complex optical assemblies.

\section{Appendix A}

The simplified cross sectional surface profile of the upper surface in the equivalent model is:

$$
f(x)=\frac{L^{2}}{2 R \pi} \sin ^{2}\left(\frac{\pi x}{L}\right), x \in\left(k L-x_{0} ; k L+x_{0}\right), \quad 2 x_{0} \leq L
$$

Where $k$ is an integer, $x_{0}$ is the length of the contacted zones, $L$ the periodic length of the

asperities (wavelength) and $h=\frac{L^{2}}{2 R \pi}$ the combine maximum height of the asperities with $R$ the mean asperities radius.

According to the contact mechanics of periodic roughness surfaces, the pressure distribution underneath the asperity inside the contacted zone can be deduced:

$$
\left.p(x)=\frac{\cos \left(\frac{\pi x}{L}\right)}{\sqrt{\sin ^{2}\left(\frac{\pi x_{0}}{L}\right)-\sin ^{2}\left(\frac{\pi x}{L}\right)}} \times\left\{\frac{E^{*} L}{4 \pi R}\left[\sin ^{2}\left(\frac{\pi x_{0}}{L}\right)-2 \sin ^{2}\left(\frac{\pi x}{L}\right)\right]+\frac{F}{L}\right\}\right)
$$

Where

$$
\mathrm{E}^{*}=\left(\frac{1-v_{1}^{2}}{\mathrm{E}_{1}}+\frac{1-v_{2}^{2}}{\mathrm{E}_{2}}\right)^{-1}
$$

With $E^{*}$ the equivalent Young's modulus, $\mathrm{E}_{1}, \mathrm{E}_{2}$ the young's modulus, $v_{1}, v_{2}$ the Poisson's ratios and $F$ is the force per asperity on the silicon surface contributed by both the adhesion 
force and the external force [14]. When $k=0$, we obtain:

$p\left(x \rightarrow x_{0}{ }^{-}\right)=\frac{-K_{I}}{\sqrt{2 \pi\left(x_{0}-x\right)}}$

Where $K_{I}$ is the stress intensity factor. Note that if we do not considering the surfaces force between contacted silicon surfaces the condition $K_{I}=0$ is satisfied and we obtain:

$F=\frac{E^{*} L^{2}}{4 \pi \mathrm{R}} \sin ^{2}\left(\frac{\pi x_{0}}{L}\right)$

When the contact is total, which means $2 x_{0} \rightarrow L$ we have:

$F_{l i m}=\frac{E^{*} L^{2}}{4 \pi R}$

For surface profile given by $\mathrm{f}(\mathrm{x})$ the energy release rate of the gap outside the contacted zone is given by:

$\zeta=\frac{K_{I}^{2}}{2 E^{*}}$

According to the JKR theory [34], the contacted zone size $2 x_{0}$ is determined by equating $\zeta$ to the surface energy $2 \omega$. Thus we have:

$F=\frac{E^{*} L^{2}}{4 \pi \mathrm{R}} \sin ^{2}\left(\frac{\pi x_{0}}{L}\right)-\sqrt{2 E^{*} L \boldsymbol{\omega} \tan \left(\frac{\pi x_{0}}{L}\right)}$

The first term corresponds to the normalized Hertz pressure and the second expresses the influence of the silicon surface force on the contact zone [25], [16], [17].

Equation (A.8) can be written as:

$\widehat{F}=\sin ^{2}(\pi c)-\alpha \sqrt{\tan (\pi c)}$ 


\section{Acknowledgements}

This work took place in the MATIOMA (Modelling and Technological improvement of Molecular Adhesion) project and has been carried out thanks to the support of the A*MIDEX project ( $\mathrm{n}^{\circ}$ ANR-11-IDEX-0001-02) funded by the « Investissements d'Avenir » French Government program, managed by the French National Research Agency (ANR). We would also like to thank the WinLight Optics Company for our technical and financial support.

\section{Bibliography}

[1] Tong Q.Y., Gösele U. Semiconductor wafer bonding: recent developments. Material Chemistry and Physics 1994; 37:101-127.

[2] Kendall K. Molecular adhesion and its applications. Kluwer Academic Publishers; 2001.

[3] Ventosa C., Rieutord F., Libralesso L., Morales C., Fournel F., Moriceau H. Hydrophilic low-temperature direct wafer bonding. Journal of Applied Physics 2008; 104:123524-1, 123524-6.

[4] Nakanishi H., Nishimoto T., Nakamura R., Yotsumoto A., Yoshida T., Shoji S. Studies on $\mathrm{SiO} 2-\mathrm{SiO} 2$ bonding with hydrofluoric acid. Room temperature and low stress binding technique for MEMS. Sensors and Actuators A, Physical 2000; 79:237-244.

[5] Berthold A., Vellekoop M. IC-compatible silicon wafer-to-wafer bonding. Sensors and Actuators, A: Physical 1997; 60:208-2011.

[6] Laurent F. MUSE Image Slicer: Test results on largest slicer ever manufactured. Proc. SPIE 7018, Advanced Optical and Mechanical Technologies in Telescopes and Instrumentation 2008; 71:7018-7030.

[7] Pamploma T. Three bipods slicer prototype: tests and finite element calculations. ProcSPIE, Advanced Optical and Mechanical Technologies in Telescopes and Instrumentation 2008; 7018:701828.1-701828.11. 
[8] Plöbl A., Kräuter G. Wafer direct bonding: tailoring adhesion between brittle materials. Material Science and Engineering 1999; R25:1-88.

[9] Liao G., Shi T., Lin X., Ma Z. Effect of surface characteristic on room-temperature silicon direct bonding, Sensor and Actuators A, Physical. 2010; 158 335-341.

[10] Yu H.H., Suo Z. A model of wafer bonding by elastic accommodation. Journal of Mechanical Physics and Solids 1998; 46(5):829-844.

[11] Gösele U. Fundamental issues on wafer bonding, in: American Society of Vacuum. 1999. [12] Gui C., Elwenspoek M. The effect of surface roughness on direct wafer bonding. Journal of Applied Physics 1999; 85:7448-7454.

[13] Miki N., Spearing S. M. Effect of nanoscale surface roughness on the bonding energy of direct-bonded silicon wafers. Journal of Applied Physics 2003; 946800.

[14] Kissinger G., Kissinger W. Void-free silicon-wafer-bond strengthening in the $200-400^{\circ} \mathrm{C}$ range, Sensors and Actuators, A: Physical 1993; 36:149-156.

[15] Stengl R., Tan T., Gösele U. A model for the silicon wafer bonding process. Japanese Journal of Applied Physics 28 1989; 10:1735-1741.

[16] Rayssac O., Olivier M., Stoemenos I., Cartier A.M., Aspar B. Silicon-on-Insulator technology and devices $\mathrm{X}$. The electrochemical society proceedings series, Pennington NJ 2001; 39:PV01-03

[17] Tang Z. Modelling the formation of spontaneous wafer direct bonding under low temperature. Microelectronic Engineering 2008; 85:1754-1757.

[18] Johnson K. The adhesion of two elastic bodies with slightly wavy surfaces. International Journal of Solids and Structures 1995; 32:423-430.

[19] Galanov B. Models of adhesive contact between rough elastic solids. International Journal of Mechanical Science 2011; 53:968-977. 
[20] Liao G., Lin X. Surface roughness modeling for silicon direct bonding. IEEE transaction on components, packaging and manufacturing technology 2011;1(8):1171 - 1177.

[21] Lai S., Lin H., Yu C. Effect of surface treatment on wafer direct bonding process. Material Chemistry and Physics 2004; 83:265-272.

[22] Klier K., Zettlemoyer A.C. Water at interface: Molecular structure and dynamics. Journal of Colloid and Interface Science 1977;58(2):216-229.

[23] Iler R. K. The chemistry of Silica. Wiley. 1979.

[24] Ventosa C., Rieutord, F., Libralesso, L., Morales, C., Fournel, F., Moriceau, H. Hydrophilic low-temperature direct wafer bonding. Journal of Applied Physics 2008; 104:123524-123526.

[25] Ventosa C., Morales C., Libralesso L., Fournel F., Papon A.M., Lafond D., Moriceau H., Penot J. D., Rieutord F. Mechanism of thermal silicon oxide direct wafer bonding. Electrochemical and Solid States Letters 2009; 12(10):375-379.

[26] Liao G. Effect of surface characteristic on room-temperature silicon direct bonding. Sensors and Actuators, A: Physical 2010; 158:335-341.

[27] Lindig O., Pannhorst W. Thermal expansion and length stability of Zerodur ${ }^{\circledR}$ in dependence on temperature and time. Applied Optics 1985; 24:3330-3334.

[28] Cocheteau N., Maurel-Pantel A., Lebon F., Rosu I., Ait Zaid S., Savin Delarclause I. Influence of process parameters on the mechanical resistance of direct bonded silica glasses. Journal of Adhesion Science and Technology 2014; 28(10):915-934.

[29] Murphy D. Fundamentals of Light Microscopy and Digital Imaging. Wiley-Liss. 2001. [30] Cocheteau N., Lebon F., Rosu I., Ait Zaid S., Savin Delarclause I. On the Modelling of the Direct Bonding of Two Silicon Surfaces. in: C.-C. Press (Ed.) Proceedings of the Eleventh International Conference on Computational Structures Technology. 2012. 
[31] Maszara W., Goetz G., Caviglia A. Bonding of silicon wafers for silicon-on insulator. Journal of Applied Physics 1988; 64:4943-4950.

[32] Cognard J. The Mechanics of the Wedge Test. The Journal of Adhesion 1986; 20:1-13.

[33] Cognard J. Use of the wedge test to estimate the lifetime of an adhesive joint in an aggressive environment. International Journal of Adhesion and Adhesives 1986; 6(4):215-220. [34] Créac'Hcadec R., Cognard J., Heuzé T. On modelling the behaviour of thin adhesive films in bonded assemblies with interface. Journal of Adhesion Science and Technology 2008; 22 (13):1541-1563.

[35] Johnson K. L., Kendall K., Roberts A. D. Surface Energy and the Contact of Elastic Solids. Proceedings of the Royal Society of London. Series A, Mathematical and Physical Sciences $1971 ; 324(1558): 301-313$

[36] Hamaker H. The London-Van der Waals attraction between spherical particles. Physica IV 1937; 10:1058-1072. 